\title{
A REMARK ON SMALL VALUES OF ENTIRE FUNCTIONS
}

\author{
by S. L. SEGAL $\dagger$
}

(Received 25th March 1966)

Let $f(z)$ be an entire function, $M(r)$ the maximum of $f(z)$ on $|z|=r$, and $\lambda>1$. Let $E_{\lambda}=E_{\lambda}(f)=\{z: \log |f(z)| \leqq(1-\lambda) \log M(|z|)\}$, and denote the density of $E_{\lambda}$ by

$$
D_{R}\left(E_{\lambda}\right)=m\left(z \in E_{\lambda}:|z| \leqq R\right) / \pi R^{2}
$$

where $m$ is planar measure.

Developing an idea of Lindelöf, Boas, Buck and Erdös (3) prove:

Theorem (A). For any $\lambda>1$, there is a positive number $K=K(\lambda)$, the same for all entire functions, such that

$$
\bar{D}\left(E_{\lambda}\right)=\varlimsup_{R \rightarrow \infty} D_{R}\left(E_{\lambda}\right) \leqq K \leqq 1 / \lambda ;
$$

Theorem (B). $\lim _{\lambda \rightarrow \infty} \lambda \underline{D}\left(E_{\lambda}\right)=\lim _{\lambda \rightarrow \infty} \lambda \varliminf_{R \rightarrow \infty} D_{R}\left(E_{\lambda}\right)=0$;

and conjecture that perhaps

also holds.

$$
\lim _{\lambda \rightarrow \infty} \lambda \bar{D}\left(E_{\lambda}\right)=0
$$

The authors of (3) do not seem to have noticed that their method gives something more, namely inequality (4) below, when the class of entire functions is restricted to those of finite positive order and type, and in fact can be used to show (in support of the conjecture $\left({ }^{*}\right)$ ) that, in certain senses, no matter how large $\lambda$ may be, $\lambda \bar{D}\left(E_{\lambda}(f)\right)$ may be arbitrarily small for proper choice of $f(z)$, where $f(z)$ has finite positive order $\rho$. (If $f$ is a polynomial $\bar{D}\left(E_{\lambda}\right)=0$.)

Precisely, we have:

Proposition I. Given $\varepsilon>0, \lambda>1$, there exists $a \rho=\rho(\varepsilon)>0$ and an entire function $f(z)$ of finite positive order $\rho$ such that

As $\varepsilon \rightarrow 0, \rho \rightarrow 0$.

$$
\lambda \bar{D}\left(E_{\lambda}(f)\right)<\varepsilon .
$$

Proposition I. Given $\varepsilon>0, \rho>0$, there exists an entire function $f(z)$ of finite positive order $\rho$ and $a \lambda=\lambda(\varepsilon)$ such that

As $\varepsilon \rightarrow 0, \lambda \rightarrow \infty$.

$$
\lambda \bar{D}\left(E_{\lambda}(f)\right)<\varepsilon
$$

$\dagger$ Fulbright Research Fellow at the University of Vienna during 1965-6. Partially supported by NSF Grant GP-5801.

$$
\text { E.M.S.-I }
$$


Both propositions follow from the same proof which is simply (3) with Jensen's Inequality replaced by Jensen's Theorem; combined with an easy and well-known estimate, and an example of Boas (1).

Proof. Let $H_{r, \lambda}=\left\{\theta: \log \left|f\left(r e^{i \theta}\right)\right| \leqq(1-\lambda) \log M(r)\right\}$. Let $f(z)$ be an entire function of finite positive order $\rho$ and finite positive type $\tau$, with $f(0)=1$, and, as usual, let $n(t)=$ the number of zeros of $f(z)$ in $|z| \leqq t$, and

Then as shown in (3),

$$
N(r)=\int_{0}^{r} n(t) / t d t
$$

$$
\begin{aligned}
\frac{1}{2 \pi} \log M(r) \int_{0}^{\infty} m\left(H_{r, \lambda}\right) d \lambda & =\log M(r)-\frac{1}{2 \pi} \int_{0}^{2 \pi} \log \mid f\left(r e^{i \theta} \mid d \theta\right. \\
& =\log M(r)-N(r),
\end{aligned}
$$

by Jensen's Theorem.

Let $l=\varliminf_{r \rightarrow \infty} n(r) / r^{\rho}$. Since $\tau$ and $\rho$ are finite, so is $l$ (e.g. (2), p. 16). Given $\eta>0$, choose $R_{0}$ such that for $r \geqq R_{0}=R_{0}(\eta)$,

(a) $M(r)>1$.

(b) $\log M(r) \leqq(\tau+\eta) r^{\rho}$.

(c) $n(r) \geqq(l-\eta) r^{\rho}$.

Then (cf. (2), p. 16, or (1), p. 28), for $r>R_{0}$,

as $r \rightarrow \infty$.

$$
N(r)=\int_{0}^{R_{0}} n(t) / t d t+\int_{R_{0}}^{r} n(t) / t d t \geqq \frac{l-\eta}{\rho} r^{\rho}+O(1),
$$

Hence, for $r>R_{0}$,

$$
\frac{N(r)}{\log M(r)} \geqq \frac{l-\eta}{\rho(\tau+\eta)}+o(1)
$$

for every $\eta>0$, as $r \rightarrow \infty$.

Also, as in (3), $m\left(z \in E_{\lambda}: R_{0} \leqq|z| \leqq R\right)=\int_{R_{0}}^{R} m\left(H_{r, \lambda}\right) r d r$.

Integrating this equation with respect to $\lambda$ and using (1) and (2) gives

$\int_{0}^{\infty} D_{R}\left(E_{\lambda}^{*}\right) d \lambda=1-R_{0}^{2} / R^{2}-\left(2 / R^{2}\right) \int_{R_{0}}^{R} \frac{r N(r)}{\log M(r)} d r \leqq 1-\frac{l-\eta}{\rho(\tau+\eta)}+o(1)$

as $R \rightarrow \infty$, for every $\eta>0$, where $E_{\lambda}^{*}=E_{\lambda} \mid\left\{z:|z| \leqq R_{0}\right\}$.

But, as observed in (3), $\lambda D_{R}\left(E_{\lambda}^{*}\right) \leqq \int_{0}^{\infty} D_{R}\left(E_{\lambda}^{*}\right) d \lambda$. Hence letting $R \rightarrow \infty$, and $\eta \rightarrow 0$, (3) becomes

$$
1-\frac{l}{\rho \tau} \geqq \lim _{R \rightarrow \infty} \lambda D_{R}\left(E_{\lambda}^{*}\right)=\lim _{R \rightarrow \infty} \lambda D_{R}\left(E_{\lambda}\right)=\lambda \bar{D}\left(E_{\lambda}\right)
$$


The inequality (4) holds for all entire functions of finite positive order and finite positive type.

Consider now the special function

$$
g_{\lambda, \rho}(z)=\prod_{n=1}^{\infty}\left\{1+z^{[\lambda]}([\lambda] n)^{-[\lambda] / p}\right\}
$$

where $[\lambda]$ is the greatest integer $\leqq \lambda$, and it is understood that if either $\rho>0$ or $\lambda>1$ is given the other is to be chosen so that $0<\rho<[\lambda]$.

As shown in (1), $g_{\lambda, \rho}(z)$ is an entire function of order $\rho$, and type

with $l=1$.

$$
(\pi /[\lambda]) \csc \pi \rho /[\lambda],
$$

Hence we have

and so the two propositions.

$$
\lambda \bar{D}\left(E_{\lambda}\left(g_{\lambda, \rho}\right)\right) \leqq 1-\frac{\sin \pi \rho /[\lambda]}{\pi \rho /[\lambda]}
$$

\section{REFERENCES}

(1) R. P. BoAs, Jr., Fundamental sets of entire functions, Annals of Math. 47 (1946), 21-32.

(2) R. P. Boas, Jr., Entire Functions (Academic Press, New York, 1954).

(3) R. P. BoAs, Jr., R. C. Buck and P. Erdös, The set on which an entire function is small, Amer. J. Math. 70 (1948), 400-402.

UNIVERSITY OF ROCHESTER

ROCHESTER, N.Y. 